\title{
The Clinical Diagnostic Values of SAA, PCT, CRP, and IL-6 in Children with Bacterial, Viral, or Co-Infections
}

\author{
Shunxin Zhu' \\ Caixiu Zeng ${ }^{2}$ \\ Yan Zou' \\ Yanjiao $\mathrm{Hu}^{\prime}$ \\ Canfang Tang' \\ Chunmei Liu ${ }^{2}$ \\ 'Clinical Laboratory, The Maternity \& \\ Child Health Care Hospital of Xiangtan \\ City, Xiangtan City, Hunan Province, \\ 4II I00, People's Republic of China; \\ ${ }^{2}$ Department of Pediatrics, The Maternity \\ \& Child Health Care Hospital of Xiangtan \\ City, Xiangtan City, Hunan Province, \\ 4III00, People's Republic of China
}

Correspondence: Shunxin Zhu Clinical Laboratory, The Maternity \& Child Health Care Hospital of Xiangtan City, Xiangtan City, Hunan Province, 4I I 100, People's Republic of China Tel +86- 18975221633

Email zhushunxin349@I26.com
Background: Bacterial, viral, and bacterial and viral co-infections generally lead to inflammatory-related diseases. The aim of this study is to assess the clinical diagnostic values of serum amyloid A (SAA), procalcitonin (PCT), C-reactive protein (CRP), and interleukin (IL)-6 in children with bacterial, viral, or co-infections.

Methods: A total of 181 children with infection symptoms (bacterial infection (Bac group), $\mathrm{n}=46$; viral infection (Vir group), $\mathrm{n}=7$; co-infections (Bac + Vir group), $\mathrm{n}=128$ ) were enrolled in our hospital from December 2019 to April 2020. Meanwhile, 42 healthy children without any infections were used as the controls. Venous blood samples were collected and the levels of serum SAA, PCT, CRP, and IL- 6 were determined by immunoluminometric assay under an $\mathrm{IMMAGE}^{\circledR}$ analyzer. The diagnostic values of these biomarkers were assessed using the receiver operating characteristic (ROC) curves.

Results: The results indicated that IL-6 level was increased in three infection groups compared to the controls. Both SAA and CRP were significantly elevated in Vir and Bac + Vir groups. High level of PCT was observed in Bac and Bac + Vir goups. In addition, PCT (0.9281, 95\% CI $=0.8645-0.9916)$ alone is an effective method for identifying bacterial infections. SAA in combination with $\mathrm{CRP}$ may distinguish co-infection from bacterial infection. Simultaneous positive of SAA, PCT, IL-6, and CRP can discriminate coinfection from the healthy controls.

Conclusion: In a word, the levels of serum SAA, PCT, CRP, and IL-6 are increased to varying degrees in different infection situations. Our findings may provide early diagnosis for patients with different infections.

Keywords: bacterial infection, viral infection, co-infection, combined biomarkers, diagnostic accuracy

\section{Introduction}

Bacterial, viral, and bacterial and viral co-infections generally lead to some inflammatory diseases, such as respiratory diseases ${ }^{1,2}$ or even septicopyemia. ${ }^{3-5}$ With the development of these inflammatory diseases caused by infections, some acute phase proteins are induced. ${ }^{3,6-8}$ For instance, procalcitonin (PCT) is a more specific and sensitive diagnosis marker for suspected bacterial infections relative to $\mathrm{C}$-reactive protein (CRP). ${ }^{6}$ PCT, CRP, and interleukin (IL)-6 can serve as diagnostic markers for septicopyemia patients induced by bacterial infections. ${ }^{3} \mathrm{~S}$ PCT combined with CRP and IL- 6 is used to identify bacterial and viral co-infection pneumonia. ${ }^{7}$ In viral infection, the level of serum amyloid A (SAA) increases more than CRP level. ${ }^{8}$ 
Because of the different concentrations (or sensitivities) of these proteins or cytokines in different infection situations, they are generally used to distinguish bacterial, viral, and co-infections in clinic. ${ }^{8-10}$ Bozlu et al uncovered that the level of serum PCT in children with fever caused by bacterial infection continues to increase within 1 week and believed that PCT may help to differentiate bacterial and viral infections. ${ }^{9}$ Generally, it is difficult to differentiate bacterial infection from viral infection in children with lower respiratory tract infections (LRTI); ${ }^{11}$ however, Zhu et al reported that PCT combined with CRP and IL-6 improves the diagnostic accuracy of LRTI. ${ }^{10}$ Perez reported that the prematurely elevated SAA is helpful to discriminate viral etiologies from bacterial etiologies. ${ }^{8}$

In this study, to our knowledge, we for the first time explored the clinical diagnostic values of SAA, PCT, CRP, and IL-6 in children with bacterial, viral, or co-infections. These findings may develop an underlying method to improve diagnostic determination for the etiologies of the above three infections.

\section{Materials and Methods}

\section{Patients and Controls}

A total of 181 children with infection symptoms were enrolled in our hospital from December 2019 to April 2020. We included only those children who were immunocompetent, who had no chronic disease, pulmonary or otherwise, and who had not received antibiotics in the 10 days before admission. Among them, 46 children were bacterial infection (Bac group), 7 cases were viral infection (Vir group), and 128 cases were co-infections (Bac + Vir group). In addition, 42 healthy children without any infections were used as the controls (HC group).

Chest $\mathrm{x}$-ray was taken on hospital admission in all children, and blood cultures were set up immediately. A throat, urine, cerebrospinal fluid, pleural fluid, joint fluid cultures were taken if necessary. All the children were divided into four groups as bacterial infection (Bac group), viral infection (Vir group), bacterial-viral coinfections (Bac + Vir group), and healthy controls (HC group). The patients in the Bac group $(n=46)$ were included according to the following criteria: 1) the signs suggesting bacterial lower respiratory tract infections in the chest radiograph (such as pleural effusion, consolidation, and lobar involvement) and 2) the presence of positive blood cultures and other culture positivity. The patients in the Vir group $(\mathrm{n}=7)$ were included as follows: 1) normal chest x-ray or the presence of patterns suggestive of viral lower respiratory tract infections (interstitial infiltrate and peribronchial infiltrates) and 2) the absence of positive blood cultures and other culture negativity. Patients in the Bac + Vir group $(\mathrm{n}=128)$ had the mixed infection symptoms. The healthy children $(n=42)$ were included as follows: 1$)$ the absence of positive blood cultures and other culture negativity and 2) without any infection symptoms. The clinicopathologic characteristics of these children were in Table 1. This study was approved by the Medical Ethics Committee of The Maternity \& Child Health Care Hospital of Xiangtan City in accordance with the Declaration of Helsinki. Meanwhile, the legal guardian of each participant signed the written informed consent.

\section{Serum Samples}

Venous blood samples $(2 \mathrm{~mL})$ from the infected children and healthy controls were collected in the morning using an evacuated tube system into a citrate-anticoagulant tube. All the blood samples were centrifuged $(2000 \mathrm{r} / \mathrm{min})$ for $10 \mathrm{~min}$ to separate the serum and stored in $-80^{\circ} \mathrm{C}$ for further experiments.

\section{Measurement for Serum Biochemical Biomarkers}

The levels of serum PCT, CRP, and SAA were determined by immunoluminometric assay under an $\mathrm{IMMAGE}^{\circledR}$ analyzer (Beckman-Coulter, Villepinte, France). The sensitivities of these assays were $0.08 \mathrm{ng} / \mathrm{mL}$ for PCT, $0.15 \mathrm{mg} / \mathrm{L}$ for CRP, and $5 \mathrm{mg} / \mathrm{L}$ for SAA. In addition, IL-6 level was measured using a commercial ELISA kit (Enzyme-linked Biotech Ltd, Shanghai, China) with a lower detection limit of $10.21 \mathrm{pg} / \mathrm{mL}$.

Table I Clinicopathologic Characteristics of the Patients and Healthy Controls

\begin{tabular}{|l|l|l|l|l|l|}
\hline Variable & Heathy Control $(\mathbf{n}=\mathbf{4 2})$ & Bacterial $(\mathbf{n = 4 6 )}$ & Virus $(\mathbf{n = 7})$ & Bacterial+Virus $(\mathbf{n = |} \mathbf{2 8})$ & $\mathbf{P}$ value \\
\hline Gender,male & $26(61.90 \%)$ & $25(52.17 \%)$ & $3(42.86 \%)$ & $73(57.03 \%)$ & 0.773 \\
Age, year & $1.69 \pm 1.20$ & $1.23 \pm 1.21$ & $1.32 \pm 0.69$ & $1.69 \pm 0.96$ & $0.07 \mid 3$ \\
\hline
\end{tabular}




\section{Statistical Analysis}

SPSS 20.0 software (IBM, Armonk, NY, USA) were used for statistical analysis and the levels of PCT, CRP, SAA, and IL- 6 were presented as mean \pm standard deviation (mean \pm SD). One-way ANOVA followed by Tukey's multiple comparisons test (homogeneity of variance) and Kruskal-Wallis test followed by Dunn's multiple comparisons test (heterogeneity of variance) were used to assess the experimental data. The receiver operating characteristic (ROC) curves were plotted to assess the clinical diagnostic values of SAA, PCT, CRP, and IL-6. P-value less than 0.05 indicated a statistically significant difference.

\section{Results}

\section{Analysis for Patient Characteristics}

We initially analyzed the clinicopathologic characteristics between infected patients and healthy controls. As shown in Table 1, 46 patients (males: 25, females: 21; mean age: $1.23 \pm$ 1.21 years) were considered as bacterial infection, 7 patients were viral infection (males: 3 , females: 4 ; mean age: $1.32 \pm$ 0.69 years), while 128 patients (males: 73, females: 55; mean age: $1.69 \pm 0.96$ years) were suffered from bacterial-viral coinfections. The above parameters for the controls were males: 26, females: 16 , and mean age: $1.69 \pm 1.20$ years.

\section{Infection Symptoms of the Patients}

Then, the distributions of disease diagnosis under different infection statuses were analyzed. As shown in Table 2, the symptom distributions of bacterial infection patients were respiratory tract infection $(\mathrm{n}=38 ; 82.61 \%)$, influenza $(\mathrm{n}=3$; $6.25 \%)$, septicopyemia $(n=2 ; 4.35 \%)$, fever $(n=1 ; 2.17 \%)$, and intestinal infection $(\mathrm{n}=2 ; 4.35 \%)$, while only respiratory tract infection $(\mathrm{n}=6 ; 85.71 \%)$ and intestinal infection

Table 2 Infection Symptoms of the Patients

\begin{tabular}{|l|c|c|c|}
\hline & $\begin{array}{l}\text { Bacterial } \\
(\mathbf{n = 4 6 )}\end{array}$ & $\begin{array}{l}\text { Virus } \\
(\mathbf{n}=\mathbf{7})\end{array}$ & $\begin{array}{l}\text { Bacterial+Virus } \\
\mathbf{( n = 1 2 8 )}\end{array}$ \\
\hline $\begin{array}{l}\text { Respiratory tract } \\
\text { infection }\end{array}$ & $38(82.61 \%)$ & 6 & $91(71.09 \%)$ \\
Influenza & $3(6.52 \%)$ & - & $8(6.25 \%)$ \\
Septicopyemia & $2(4.35 \%)$ & - & $5(3.91 \%)$ \\
Fever & $1(2.17 \%)$ & - & $2(1.56 \%)$ \\
Intestinal infection & $2(4.35 \%)$ & 1 & $5(3.91 \%)$ \\
Ballismus & - & $(14.29 \%)$ & $3(2.34 \%)$ \\
Convulsion & - & - & $7(5.47 \%)$ \\
Others & - & - & $7(5.47 \%)$ \\
\hline
\end{tabular}

$(\mathrm{n}=1 ; 14.29 \%)$ were found in viral infection patients. Shockingly, the symptoms of co-infections were the most widely distributed, including espiratory tract infection $(\mathrm{n}=$ $91 ; 71.09 \%)$, influenza $(n=8 ; 6.25 \%)$, septicopyemia $(n=5$; $3.91 \%)$, fever $(\mathrm{n}=2 ; 1.56 \%)$, intestinal infection $(\mathrm{n}=5$; $3.91 \%)$, ballismus $(\mathrm{n}=3 ; 2.34 \%)$, convulsion $(\mathrm{n}=7$; $5.47 \%)$, and others $(\mathrm{n}=7 ; 5.47 \%)$.

\section{Analysis for the Levels of Serum Biochemical Biomarkers in Children with Bacterial, Viral, or Co-Infections}

Afterwards, the levels of SAA, PCT, IL-6, and CRP in children with bacterial, viral, or co-infections were assessed. The analysis results for Figure 1 were as follows:

SAA: There were significant differences between Vir group and $\mathrm{HC}$ group (Figure $1 \mathrm{~A}, P<0.01$ ) as well as between $\mathrm{Bac}+$ Vir group and HC group $(P<0.001)$. In addition, compared to the Bac group, increased level of SAA was observed in both the Vir group $(P<0.01)$ and Bac + Vir group $(P<0.001)$. Interestingly, there seems to be no significant differences between the Bac and HC groups.

PCT: Similarly, PCT level was also remarkably elevated in Bac + Vir group relative to the HC group (Figure 1B). Significant differences were also existed between $\mathrm{Bac}+\mathrm{Vir}$ group and Bac group $(P<0.001)$ or Vir group $(P<0.001)$. Distinguished from SAA level, the level of PCT was significantly increased in the Bac group compared to that of the HC group $(P<0.001)$; however, no significant difference was found between the Vir group and HC group.

IL-6: In comparison to the HC group, we found that IL-6 level was significantly elevated in the Bac (Figure $1 \mathrm{C}, P<0.01)$, Vir $(P<0.001)$, and $\mathrm{Bac}+\mathrm{Vir}$ groups $(P<0.001)$. Furthermore, the level of IL-6 was further increased in $\mathrm{Bac}+\mathrm{Vir}$ group compared with the Bac group $(P<0.001)$.

CRP: As shown in Figure 1D, the results of serum CRP level in children with bacterial, viral, or co-infections were similar to that of IL-6 except that no significant difference was found between the Bac and $\mathrm{HC}$ groups.

\section{Diagnostic Values of Serum Biochemical Biomarkers in Children with Bacterial, Viral, or Co-Infections}

ROC curve analysis was used for the assessment of the diagnostic values of SAA, PCT, IL-6, and CRP in children with bacterial, viral, or co-infections. First of all, there were relatively few cases in the Vir group, and therefore ROC curve 

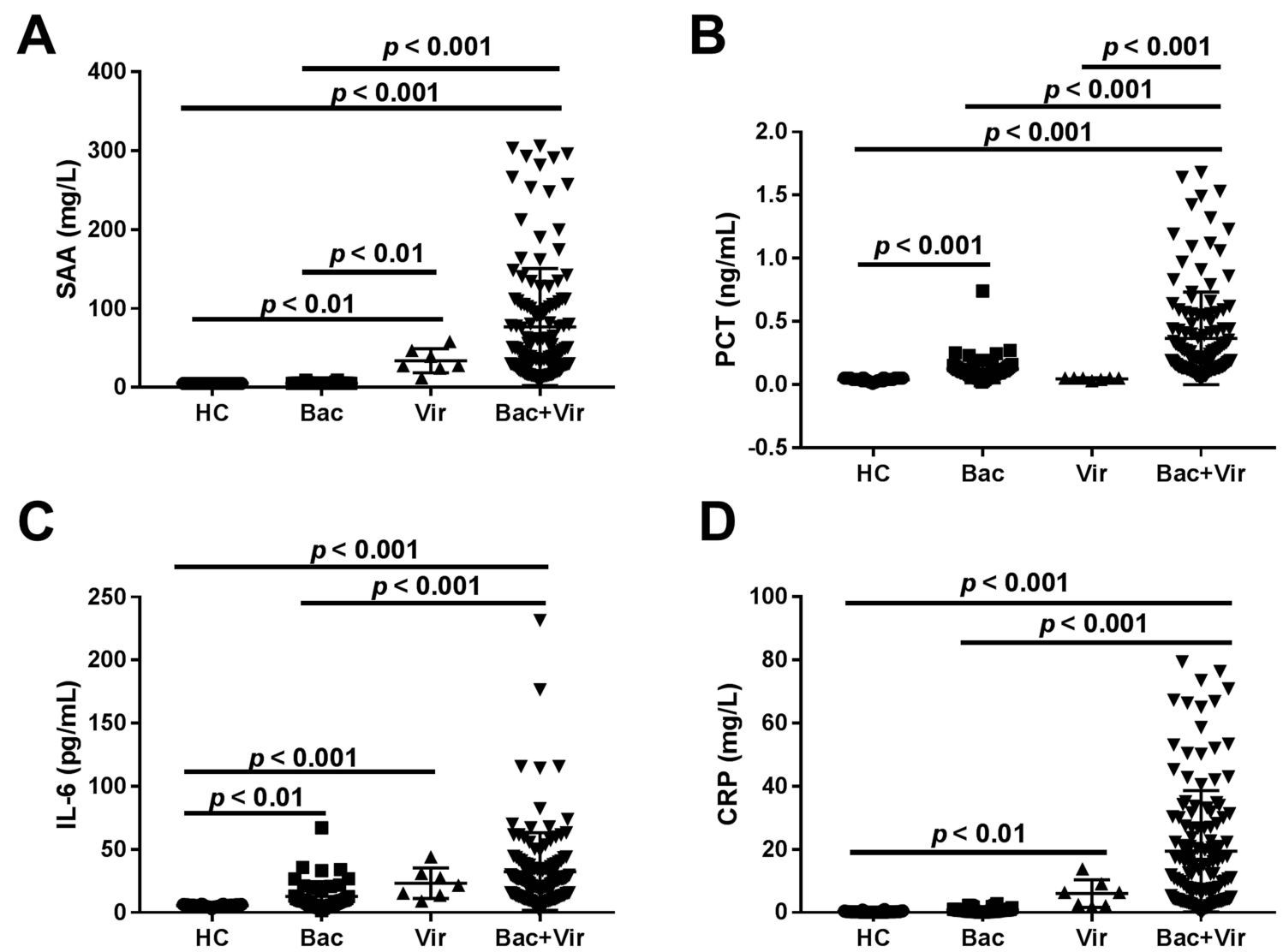

D

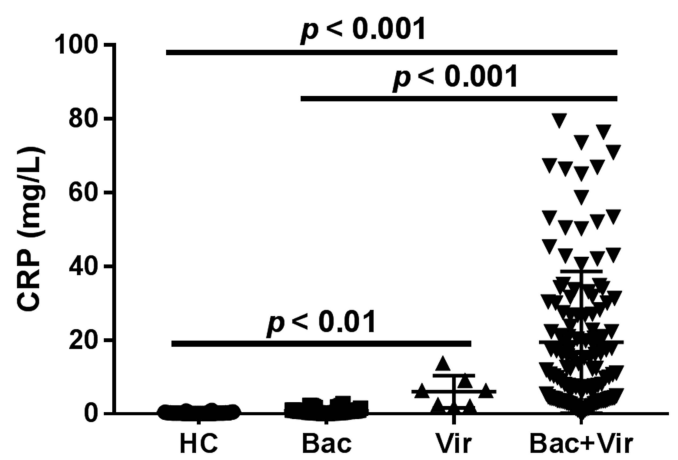

Figure I Analysis for the levels of serum biochemical biomarkers in children with bacterial, viral, or co-infections. (A) The level of serum SAA in children with bacterial, viral, or co-infections. (B) The level of serum PCT in children with bacterial, viral, or co-infections. (C) The level of serum IL-6 in children with bacterial, viral, or coinfections. (D) The level of serum CRP in children with bacterial, viral, or co-infections.

analysis was not carried out. However, the levels of SAA and IL-6 in seven patients with viral infection were remarkably higher than those of controls. Meanwhile, no significant differences were found in the results of PCT level. Only one case had a significant increase in CRP level (data were not shown). As illustrated in Figure 2A and Table 3, SAA cannot distinguish Bac from $\mathrm{HC}(\mathrm{AUC}=0.5417$, sensitivity $=8.33 \%$, specificity $=100 \%, 95 \% \mathrm{CI}=0.4225-0.6608$ ) completely. Compared to $\mathrm{CRP}$ (AUC $=0.8383$, sensitivity $=65.22 \%$, specificity $=83.33 \%, 95 \% \mathrm{CI}=0.7558-0.9207)$ and IL-6 $(\mathrm{AUC}=0.7751$, sensitivity $=69.57 \%$, specificity $=78.57 \%$, $95 \% \mathrm{CI}=0.6647-0.8828)$, PCT $(\mathrm{AUC}=0.9281$, sensitivity $=$ $86.96 \%$, specificity $=100 \%, 95 \% \mathrm{CI}=0.8645-0.9916$ ) can distinguish Bac from $\mathrm{HC}$ to some extent. In terms of the diagnostic values of these serum biochemical indexes in coinfection (Figure 2B and Table 3), we demonstrated that SAA $(\mathrm{AUC}=1$, sensitivity $=100 \%$, specificity $=100 \%, 95 \% \mathrm{CI}=$ $1-1)$, PCT $($ AUC $=0.9961$, sensitivity $=99.22 \%$, specificity $=$
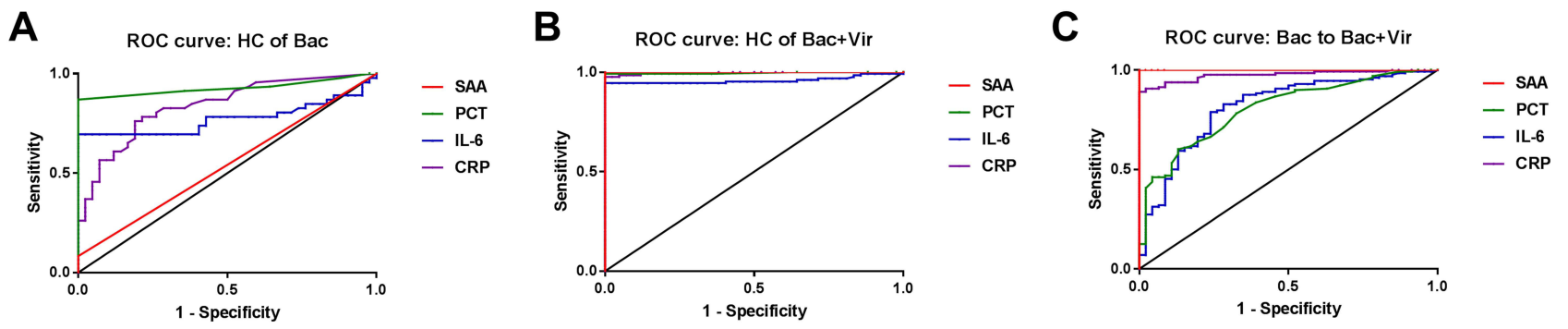

Figure 2 Diagnostic values of serum biochemical biomarkers in children with bacterial, viral, or co-infections. (A) ROC curves for SAA, PCT, IL-6, and CRP for discrimination between bacterial infection and healthy control. (B) ROC curves for SAA, PCT, IL-6, and CRP for discrimination between co-infection and healthy control. (C) ROC curves for SAA, PCT, IL-6, and CRP for discrimination between co-infection and bacterial infection. 
Table 3 Sensitivity and Specificity of SAA, PCT, CRP, and IL-6 in Different Groups

\begin{tabular}{|c|c|c|c|c|c|c|c|}
\hline Group & Biomarker & Cut-off & AUC & Sensitivity (\%) & Specificity (\%) & $95 \% \mathrm{Cl}$ & $P$ value \\
\hline \multirow[t]{4}{*}{$\mathrm{HC}$ of $\mathrm{Bac}$} & SAA & $>5.56$ & 0.5417 & 8.33 & 100 & $0.4225-0.6608$ & 0.4969 \\
\hline & РСT & $>0.055$ & $0.928 I$ & 86.96 & 100 & $0.8645-0.9916$ & $<0.0001$ \\
\hline & CRP & $>0.405$ & 0.8383 & 65.22 & 83.33 & $0.7558-0.9207$ & $<0.0001$ \\
\hline & IL-6 & $>6.18$ & 0.7751 & 69.57 & 78.57 & $0.6647-0.8828$ & $<0.0001$ \\
\hline \multirow[t]{4}{*}{$\mathrm{HC}$ of $\mathrm{Bac}+\mathrm{Vir}$} & SAA & $>7.53$ & 1 & 100 & 100 & $1-1$ & $<0.0001$ \\
\hline & РCT & $>0.055$ & 0.9961 & 99.22 & 100 & $0.9882-1.004$ & $<0.0001$ \\
\hline & CRP & $>1.025$ & 0.9958 & 97.66 & 100 & $0.9894-1.002$ & $<0.0001$ \\
\hline & IL-6 & $>6.42$ & 0.9588 & 94.53 & 95.24 & $0.9278-0.9898$ & $<0.0001$ \\
\hline \multirow[t]{4}{*}{$\mathrm{Bac}$ to $\mathrm{Bac}+\mathrm{Vir}$} & SAA & $>10.13$ & I & 100 & 100 & $1-1$ & $<0.0001$ \\
\hline & РCT & $>0.155$ & 0.8060 & 64.06 & 80.43 & $0.7365-0.8756$ & $<0.0001$ \\
\hline & CRP & $>2.465$ & 0.9745 & 90.63 & 97.83 & $0.9545-0.9946$ & $<0.0001$ \\
\hline & IL-6 & $>18.02$ & 0.8145 & 67.97 & 78.26 & $0.7405-0.8885$ & $<0.0001$ \\
\hline
\end{tabular}

Abbreviations: $\mathrm{HC}$, healthy control; Bac, bacterial infection; Bac + Vir, bacterial and viral co-infections; SAA, serum amyloid A; PCT, procalcitonin; CRP, C-reactive protein; IL-6, interleukin-6.

$100 \%, 95 \%$ CI $=0.9882-1.004)$, CRP $(\mathrm{AUC}=0.9958$, sensitivity $=97.66 \%$, specificity $=100 \%, 95 \% \mathrm{CI}=0.9894-1.002)$, and IL-6 (AUC $=0.9588$, sensitivity $=94.53 \%$, specificity $=$ 95.24\%, 95\% CI $=0.9278-0.9898$ ) can distinguish coinfection from HC. Interestingly, we further revealed that both PCT (AUC $=0.8060$, sensitivity $=64.06 \%$, specificity $=80.43 \%, 95 \% \mathrm{CI}=0.7365-0.8756)$ and IL-6 (AUC $=$ 0.8145 , sensitivity $=67.97 \%$, specificity $=78.26 \%, 95 \%$ CI $=0.7405-0.8885$ ) cannot distinguish co-infection from Bac. On the contrary, SAA (AUC $=1$, sensitivity $=100 \%$, specificity $=100 \%, 95 \% \mathrm{CI}=1-1)$ and $\mathrm{CRP}(\mathrm{AUC}=0.9745$, sensitivity $=90.63 \%$, specificity $=97.83 \%$, 95\% CI $=$ 0.9545-0.9946) can distinguish co-infection from Bac (Figure 2C and Table 3).

\section{Discussion}

Bacterial, viral, and co-infections generally result in severe diseases in the respiratory system $^{1,2}$ or even septicopyemia. $^{3,12,13}$ Therefore, effective biomarkers to distinguish infection states can provide targeted treatments for these diseases. Currently, many acute phase proteins or cytokines have been widely used in the clinical diagnosis for infections, such as SAA, PCT, IL-6, and CRP. ${ }^{3,6-10}$ However, to our knowledge, these biomarkers are only used to discriminate two of these infections in numerously previous studies. Besides, the application of only one biomarker (may be two or three) is still not discriminatory enough to indicate infection situations due to the relatively poor sensitivity and specificity. ${ }^{14-16}$ Therefore, enhancing the sensitivity and specificity on the estimation of classification of infection is necessary.
In the current study, we included the proven biomarkers of SAA, PCT, CRP, and IL-6 and mainly explored their clinical diagnostic values in children with bacterial, viral, or co-infections. We initially determined the levels of these biomarkers in the Bac, Vir, Bac + Vir, and control groups. In this study, increased SAA and CRP were observed in Vir group compared to that of control. Similarly, a recent study focused on coronavirus has uncovered that the levels of SAA and CRP were significantly elevated in COVID-19 patients and were continually increased with the aggravation of the disease. ${ }^{17} \mathrm{We}$ speculated the increasing SAA and CRP may be associated with viral infection-related diseases. In addition, we further demonstrated that both SAA and CRP were also significantly increased in co-infection children. Combined the above data, we believed that the enhancements of SAA and CRP may be valuable information for viral infection and co-infection diagnosis. Further data demonstrated that compared to the controls, IL-6 level was increased in bacterial infection, viral infection, and co-infection patients. Interestingly, the results of some previous studies are consistent with our findings. For example, $\mathrm{Wu}$ et al conducted a meta-analysis for serum IL-6 in bacterial infection of cirrhotic patients and found an obviously increased IL-6 in these patients. ${ }^{18}$ Copaescu et al believed that severe acute respiratory syndrome coronavirus 2 (SARS-CoV-2) can significantly promote the secretion of IL-6. ${ }^{19}$ Based on these data, we speculated that IL-6 may be a ubiquitous cytokine in infection diseases and have no specificity for the diagnosis of different infections. For PCT, as the previous studies mentioned, ${ }^{20,21}$ also high level of PCT was found 
in bacterial infection children. Additionally, we also determined a higher PCT level in co-infection subjects. We speculated that PCT is an efficient biomarker for both bacterial infection and co-infection diagnosis.

Given that the biomarkers were increased in these infection situations at the same time, we further investigated the diagnostic values of SAA, CRP, IL-6, and PCT for discrimination different infections using ROC curve models. As presented in Figure 2A and Table 3, PCT has the largest area of AUC among the four biomarkers. We speculated that compared to CRP, IL-6, and SAA, PCT is a better biomarker for distinguishing Bac from HC. In terms of the diagnostic values of these serum biochemical biomarkers in co-infection, SAA, PCT, IL-6, and CRP all had relatively large AUC area and high sensitivity/specificity. We believed that the combined use of SAA, PCT, IL-6, and CRP (simultaneous positive) is more likely to indicate co-infection. Afterwards, to distinguish co-infection from bacterial infection, we found that the AUC, sensitivity, and specificity of SAA and CRP were higher compared to those of PCT and IL-6, suggesting that combination analysis of SAA and CRP facilitate distinguishing between bacterial and coinfections. In addition, ROC curve was not performed in the Vir group due to the few viral infection cases in this study. It may be a limitation of this study and we will consider large size samples in future studies.

\section{Conclusion}

The levels of SAA, CRP, IL-6, and PCT are increased in different infection situations. Furthermore, the utilization of PCT as a single biomarker is an effective method to obtain a better prediction for identification bacterial infection. Meanwhile, the analysis of SAA in combination with CRP may distinguish co-infection from bacterial infection. More importantly, we found that simultaneous positive of SAA, PCT, IL-6, and CRP is enough to discriminate co-infection from the healthy controls. In a word, combined laboratory measurements of SAA, PCT, IL-6, and CRP can not only provide early diagnosis for patients with different infections but also enhance the sensitivity and specificity on the estimation of classification of infection, which is of great prognostic value.

\section{Data Sharing Statement}

All data generated or analyzed during this study are included in this published article.

\section{Ethics Approval and Informed Consent}

This study was approved by the Medical Ethics Committee of The Maternity \& Child Health Care Hospital of Xiangtan City in accordance with the Declaration of Helsinki. Meanwhile, the legal guardian of each participant signed the written informed consent.

\section{Author Contributions}

All authors made substantial contributions to conception and design, acquisition of data, or analysis and interpretation of data; took part in drafting the article or revising it critically for important intellectual content; gave final approval of the version to be published; and agree to be accountable for all aspects of the work.

\section{Funding}

No funding was received.

\section{Disclosure}

The authors declare that they have no competing interests.

\section{References}

1. Langford BJ, So M, Raybardhan S, et al. Bacterial co-infection and secondary infection in patients with COVID-19: a living rapid review and meta-analysis. Clin Microbiol Infect. 2020;26(12):1622-1629. doi:10.1016/j.cmi.2020.07.016

2. Rowe HM, Meliopoulos VA, Iverson A, Bomme P, Schultz-Cherry S, Rosch JW. Direct interactions with influenza promote bacterial adherence during respiratory infections. Nat Microbiol. 2019;4 (8):1328-1336. doi:10.1038/s41564-019-0447-0

3. Gao L, Liu X, Zhang D, et al. Early diagnosis of bacterial infection in patients with septicopyemia by laboratory analysis of PCT, CRP and IL-6. Exp Ther Med. 2017;13(6):3479-3483. doi:10.3892/ etm. 2017.4417

4. Lin HY. The severe COVID-19: a sepsis induced by viral infection? And its immunomodulatory therapy. Chin J Traumatol. 2020;23 (4):190-195. doi:10.1016/j.cjtee.2020.06.002

5. Richter DC, Heininger A, Brenner T, et al. [Bacterial sepsis: diagnostics and calculated antibiotic therapy]. Anaesthesist. 2017;66 (10):737-761. German. doi:10.1007/s00101-017-0363-8

6. Simon L, Gauvin F, Amre DK, Saint-Louis P, Lacroix J. Serum procalcitonin and C-reactive protein levels as markers of bacterial infection: a systematic review and meta-analysis. Clin Infect Dis. 2004;39(2):206-217. doi:10.1086/421997

7. Do Q, Dao TM, Nguyen TNT, Tran QA, Nguyen HT, Ngo TT. Procalcitonin identifies bacterial coinfections in Vietnamese children with severe respiratory syncytial virus pneumonia. Biomed Res Int. 2020;2020:7915158. doi:10.1155/2020/7915158

8. Perez L. Acute phase protein response to viral infection and vaccination. Arch Biochem Biophys. 2019;671:196-202. doi:10.1016/ j.abb.2019.07.013

9. Bozlu G, Tanriverdi H, Aslan G, Kuyucu N. The value of acute phase reactants and LightCycler(R) SeptiFast test in the diagnosis of bacterial and viral infections in pediatric patients. Arch Argent Pediatr. 2018;116(1):35-41. 
10. Zhu G, Zhu J, Song L, Cai W, Wang J. Combined use of biomarkers for distinguishing between bacterial and viral etiologies in pediatric lower respiratory tract infections. Infect Dis. 2015;47(5):289-293. doi:10.3109/00365548.2014.987163

11. Bruyndonckx R, Stuart B, Little P, et al. Amoxicillin for acute lower respiratory tract infection in primary care: subgroup analysis by bacterial and viral aetiology. Clin Microbiol Infect. 2018;24 (8):871-876. doi:10.1016/j.cmi.2017.10.032

12. Lin GL, McGinley JP, Drysdale SB, Pollard AJ. Epidemiology and immune pathogenesis of viral sepsis. Front Immunol. 2018;9:2147.

13. Srinivasan V, Mohamed M, Kato H. Melatonin in bacterial and viral infections with focus on sepsis: a review. Recent Pat Endocr Metab Immune Drug Discov. 2012;6(1):30-39. doi:10.2174/ 187221412799015317

14. Ten Oever J, Tromp M, Bleeker-Rovers CP, et al. Combination of biomarkers for the discrimination between bacterial and viral lower respiratory tract infections. $J$ Infect. 2012;65(6):490-495. doi:10.1016/j.jinf.2012.08.004

15. Jiyong J, Tiancha $\mathrm{H}$, Wei $\mathrm{C}$, Huahao S. Diagnostic value of the soluble triggering receptor expressed on myeloid cells- 1 in bacterial infection: a meta-analysis. Intensive Care Med. 2009;35(4):587-595. doi:10.1007/s00134-008-1333-z
16. Kruger S, Ewig S, Papassotiriou J, et al. Inflammatory parameters predict etiologic patterns but do not allow for individual prediction of etiology in patients with CAP: results from the German competence network CAPNETZ. Respir Res. 2009;10:65. doi:10.1186/1465-9921-10-65

17. Li H, Xiang X, Ren H, et al. Serum amyloid A is a biomarker of severe coronavirus disease and poor prognosis. J Infect. 2020;80 (6):646-655. doi:10.1016/j.jinf.2020.03.035

18. Wu Y, Wang M, Zhu Y, Lin S. Serum interleukin-6 in the diagnosis of bacterial infection in cirrhotic patients: a meta-analysis. Medicine. 2016;95(41):e5127. doi:10.1097/MD.0000000000005127

19. Copaescu A, Smibert O, Gibson A, Phillips EJ, Trubiano JA. The role of IL-6 and other mediators in the cytokine storm associated with SARS-CoV-2 infection. J Allergy Clin Immunol. 2020;146(3):518534 e511. doi:10.1016/j.jaci.2020.07.001

20. Memar MY, Varshochi M, Shokouhi B, Asgharzadeh M, Kafil HS Procalcitonin: the marker of pediatric bacterial infection. Biomed Pharmacother. 2017;96:936-943. doi:10.1016/j.biopha.2017.11.149

21. Hu L, Shi Q, Shi M, Liu R, Wang C. Diagnostic value of PCT and CRP for detecting serious bacterial infections in patients with fever of unknown origin: a systematic review and meta-analysis. Appl Immunohistochem Mol Morphol. 2017;25(8):e61-e69. doi:10.1097/ PAI.0000000000000552
International Journal of General Medicine

\section{Publish your work in this journal}

The International Journal of General Medicine is an international, peer-reviewed open-access journal that focuses on general and internal medicine, pathogenesis, epidemiology, diagnosis, monitoring and treatment protocols. The journal is characterized by the rapid reporting of reviews, original research and clinical studies

\section{Dovepress}

across all disease areas. The manuscript management system is completely online and includes a very quick and fair peer-review system, which is all easy to use. Visit http://www.dovepress.com/ testimonials.php to read real quotes from published authors. 